Pacific Journal of Mathematic

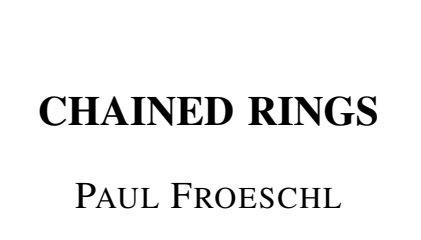




\title{
CHAINED RINGS
}

\author{
Paul A. Froeschl III
}

\begin{abstract}
All rings considered are commutative with identity. A chained ring is any ring whose set of ideals is totally ordered by inclusion. The main object of this paper is stating conditions in which every valuation overring of a given ring is a chained ring. It is shown that every valuation overring of a ring $R$ is a chained ring if and only if the ideal of zero divisors of $T(R)$, the total quotient ring of $R$, is the conductor of $\underline{R}$, the integral closure of $R$, in $T(R)$. An example is provided of a valuation ring which is not a chained ring even though its total quotient ring is a chained ring.
\end{abstract}

1. Preliminaries. In this short section we set notation and establish some elementary results.

A total quotient ring is a ring consisting entirely of zero divisors and units. If $R$ is a ring, then let $T(R)$ denote the smallest total quotient ring containing $R$. We will call $T(R)$ the total quotient ring of $R$.

Let $G$ be a totally ordered abelian group. A valuation on a ring $T$ is a function $v$ from $T$ onto $G \cup\{\infty\}$, such that for all $x$ and $y$ in $T$ :

(1) $\quad v(x y)=v(x)+v(y)$;

(2) $v(x+y) \geqq \min \{v(x), v(y)\}$;

(3) $v(1)=0$ and $v(0)=\infty$.

Let $V$ be a ring, let $P$ be a prime ideal of $V$, and let $T$ be a ring containing $V$. Manis [11] defined the pair $(V, P)$ to be a valuation pair of $T$ if the following equivalent conditions are satisfied:

(a) If $S$ is a subring of $T$ containing $V$ and if $M$ is a prime ideal of $S$ such that $M \cap V=P$, then $V=S$;

(b) For each $x$ in $T \backslash V$, there exists $y$ in $P$ such that $x y$ is in $V \backslash P$;

(c) There is a valuation $(v, G)$ on $T$ such that

$$
V=\{x \in T \mid v(x) \geqq 0\} \quad \text { and } \quad P=\{x \in T \mid v(x)>0\}
$$

If $(V, P)$ is a valuation pair of $T$, then $V$ is called a valuation ring. We will sometimes represent $V$ by $T_{v}$. In this paper we always assume that $T$ is $T(V)$. Chained rings are valuation rings, and in the domain case the concepts are equivalent to the concept of a valuation domain.

When we deal with rings containing zero divisors, we say that 
an ideal is regular provided it contains a regular element, a nonzero divisor. We therefore define a Prüfer ring as a ring in which every finitely generated regular ideal is invertible. Fourteen equivalent definitions for a Prüfer ring were given by Griffin [7]. Moreover, examples by Gilmer [4] and Boisen and Larsen [1] show that a valuation ring need not be a Prüfer ring. Consequently, we define a Prüfer valuation ring as a valuation ring that is also a Prüfer ring.

A ring $R$ has few zero divisors if the set of zero divisors of $R$ is a finite union of prime ideals. A ring $R$ is additively regular if for each $x$ in $T(R)$ there exists a $u$ in $R$ such that $x+u$ is a regular element of $T(R)$. Davis [3] showed that a ring with few zero divisors is additively regular. Gilmer and Huckaba [6] have shown that the converse is not true. In his paper Davis defined what he called a quasi-valuation ring as a ring $R$ with few zero divisors such that if $x$ and $y$ are in $R$, both regular, then $x$ divides $y$ or $y$ divides $x$ in $R$. However, Griffin [7] showed that if $R$ is an additively regular ring, then $R$ is a valuation ring if and only if $R$ is a quasivaluation ring. But then Huckaba [8] established that an additively regular valuation ring is a Prüfer ring. Consequently, we define $R$ to be a quasi-valuation ring if $x$ and $y$ in $R$, both regular, implies that $x$ divides $y$ or $y$ divides $x$ in $R$.

2. Structure theorems for chained rings. If $R$ is a chained ring, then obviously any homomorphic image of $R$ is also a chained ring. Consequently, the homomorphic image of a valuation domain is a chained ring. By making use of recent results of Hungerford [9] and Boisen and Larsen [2], we are able to characterize a chained ring with Noetherian total quotient ring:

THEOREM 1. Let $R$ be a chained ring whose total quotient ring is a Noetherian ring. Then $R$ is the homomorphic image of a valuation domain.

Proof. Since $R$ is a chained ring with $T(R)$ a Noetherian ring, then it is easy to see that $T(R)$ is a principal ideal ring and satisfies the descending chain condition. Hungerford [9] has shown that $T(R)$ is the homomorphic image of a valuation domain. Indeed, $T(R)$ is the homomorphic image of a Prüfer domain. However, since $R$ is a Prüfer ring, Boisen and Larsen [2] have shown that $R$ is the homomorphic image of a Prüfer domain, say $D$. Let $\pi$ be the desired homomorphism and let $M$ be the unique maximal ideal of $R$. There is an ideal $M^{\prime}$ of $D$ such that $\pi\left(M^{\prime}\right)=M$. If $b \notin M^{\prime}$, then $\pi(b) \notin M$, and hence $\pi(b)$ is a unit of $R$. The function defined by 
$\pi^{*}: D_{M^{\prime}} \rightarrow R$ such that $\pi^{*}(a / b)=\pi(a) \pi(b)^{-1}$ is an epimorphism. Therefore, $R$ is the homomorphic image of the valuation domain $D_{M^{\prime}}$.

The question remains open as to whether every chained ring can be realized as the homomorphic image of a valuation domain.

Let $V$ be an arbitrary valuation ring with corresponding valuation $v$. If $z$ is a zero divisor of $V$, then nothing can be determined about the image of $z$ under $v$. This is not the case in chained rings, as our next result shows. It is basic to what follows.

THEOREM 2. (1) Every chained ring is a valuation ring of its total quotient ring.

(2) If $v$ is a valuation on $T$ and $T_{v}$ is chained, then $Z(T)$ is a maximal ideal of $T$ and there is a valuation $v^{\prime}$ on the field $T / Z(T)$ such that $v$ is given by $v(x)=v^{\prime}(x+Z(T))$ for all $x$ in T. Moreover, in this case $Z\left(T_{v}\right)=\{x \in T \mid v(x)=\infty\}$.

Proof. For (1) let $v^{\prime}$ be the valuation of $R_{P} / P_{P}$ whose valuation ring is $R / P$, where $P$ is the prime ideal of zero divisors of the chained ring $R$. One sees easily that $R=T(R)_{u}$, where $u$ is given by the formula $u(a / s)=v^{\prime}(a+P)-v^{\prime}(s+P)$, where $a$ and $s$ are in $R$ and $s$ is regular.

For the proof of (2), suppose $R=T_{v}$ is chained and $P$ the prime ideal of zero divisors of $R$, so that $T=R_{P}$. Then every element of $R_{P}$ either belongs to $R$ or has the form $1 / s$ for some $s$ in $R \backslash P$. If $R=T(R)$, the result is straightforward. Otherwise, we have $v(1 / s)<0$ for some regular $s$ in $R$. But then if $r$ is an element of $Z(R)$ and $v(r)=0$, we have $r R \cong s R$ so that $v(s)=0$ for all regular $s$, a contradiction. Hence, if $r$ is an element of $Z(R), v(r)>0$. If $v(r)<\infty$, there exists $s$ regular in $R$ such that

$$
v(1 / s)=-v(r) \text {, i.e. } v(r / s)=0 \text {. }
$$

But then $r=r^{\prime}$ s and $r^{\prime}$ is a zero divisor for which $v\left(r^{\prime}\right)=0$, a contradiction. This proves the "moreover" of (2). Now define a valuation of $T / Z(T)$ by $v^{\prime}(x+Z(T))=v(x)$. This is easily seen to be well defined, etc., and $Z(T)=P_{P}$ is maximal in $T$; clearly $v$ is equivalent to $u$, where $u$ is defined as in (1).

We now prove our first structure theorem:

TheOrem 3. Let $T$ be a chained total quotient ring and $V$ a valuation ring of $T$. Then $V$ is a chained ring iff $Z(T) \subseteq V$, in which case $V$ is quasilocal. Otherwise, $V$ has exactly two maximal ideals, namely $Z(V)$ and a maximal regular ideal. 
Proof. If $V$ is chained, then $x$ is an element of $Z(T)$ if and only if $x=a / s$ with $a$ and $s$ elements of $V$ and $s$ regular, $a$ an element of $Z(V)$. But clearly $a V \subseteq s V$, so $a / s$ is an element of $V$. Conversely, if $Z(T) \subseteq V$, then for all $\alpha$ in $Z(V)$ and regular $s$ in $V, a / s$ is in $V$. Now let $x$ and $y$ be in $V$, say $x T \subseteq y T$, i.e. $x=y(a / s)$ for $a$ and $s$ in $V, s$ regular. If $a$ is regular, then $v(a / s)$ or $v(s / a)$ is $\geqq 0$, i.e. $a / s$ or $s / a$ is in $V$. Hence, $x V \subseteq y V$ or $y V \subseteq x V$. If $a$ is a zero divisor, then $x$ is in $y V$. In either case, $x V$ and $y V$ are comparable.

Finally, if $Z(T) \nsubseteq V$, then there is a regular nonunit $s$ in $V$ and zero divisor such that $a / s$ is not in $V$, i.e. $a V \nsubseteq s V$. So let $M$ be the unique maximal ideal containing $s V$. [Regular primes are comparable by an argument of Davis [3]: if $M$ and $N$ are incomparable such ideals, then $M \nsubseteq N \cup Z(V)$, since $Z(V)=Z(T) \cap V$ is a prime ideal, whence there is a regular element $x$ in $M \backslash N$, and symmetrically, regular $y$ in $N \backslash M$. Since $x$ and $y$ are comparable, this is impossible.] and $N$ a maximal ideal containing $a V$. Since $N \neq M, N$ consists of zero divisors, so $N=Z(V)$, as required.

This theorem tells us precisely when a valuation ring with chained total quotient ring is or is not a chained ring. It rests completely on whether the zero divisors of the total quotient ring are or are not contained in the ring. Any homomorphic image of a valuation domain satisfies the first part of Theorem 3 . We now construct an example which show the second part of Theorem 3 is realizable; that is, we construct a chained total quotient ring $T$ that admits a valuation ring $V$ such that $T(V)=T$, but $V$ is not a chained ring.

EXAMPLE 1. Let $K$ be a Prufer $2^{\infty}$-group [2-primary part of the group $\boldsymbol{Q} / \boldsymbol{Z}$ ]. Then $K$ is a $\boldsymbol{Z}_{(2)}$-module in a natural way. Let $T=K+\boldsymbol{Z}_{(2)}$ be the idealization of the $\boldsymbol{Z}_{(2)}$-module $K$ [addition is coordinate-wise and multiplication is $\left(k_{1}+z_{1}\right)\left(k_{2}+z_{2}\right)=\left(k_{1} z_{2}+k_{2} z_{1}\right)+$ $z_{1} z_{2}$ ]. The divisibility of $K$ shows that the ideals of $T$ are exactly the subgroups of $K$ and ideals of the form $K+J, J$ an ideal of $\boldsymbol{Z}_{(2)}$. Hence $T$ is a chained ring and in fact $Z(T)=K+(2)$, so $T$ is a total quotient ring. Define a valuation on $T$ by $v(k+z)=v^{\prime}(z)$, where $v^{\prime}$ is the 3 -adic valuation of $\boldsymbol{Q}$. Then $T_{v}=K+\left(\boldsymbol{Z}_{(2)} \cap \boldsymbol{Z}_{(3)}\right)$, clearly, and $\boldsymbol{Z}_{(2)} \cap \boldsymbol{Z}_{(3)}=\boldsymbol{Z}_{(2) \cup(3)}$ is not a quasilocal ring. Since it is a homomorphic image of $T_{v}, T_{v}$ is not a chained ring, yet the total quotient ring of $T_{v}$ is $T$ itself. Incidentally, in this case $Z(T)=K+(2)$ and $K+(3)$ are the two maximal ideals of $T_{v}$.

We now list three immediate corollaries to Theorem 3 . 
CoROLlaRY 1. Let $T$ be a chained total quotient ring and $V$ a proper valuation ring of $T$ such that $T=T(V)$.

(a) If $V$ has exactly two maximal ideals, then every overring of $V$, other that $T$, is a valuation ring with exactly two maximal ideals;

(b) If $V$ is a chained ring, then every overring of $V$ is chained ring.

COROLLARY 2. Let $R$ be a ring such that $T(R)$ is a chained ring. Let $\left\{V_{a}\right\}$ be a set of valuation rings, totally ordered by inclusion, between $R$ and $T(R)$. If one member of $\left\{V_{a}\right\}$ is a chained ring, then every member of $\left\{V_{a}\right\}$ is a chained ring. If one member of $\left\{V_{a}\right\}$ has exactly two maximal ideals, then every member of $\left\{V_{a}\right\}$ has exactly two maximal ideals.

COROLLARY 3. Every overring of a chained ring is a chained ring.

We have seen by Example 1 that there exist chained total quotient rings $T$ that admit valuation rings which are not chained rings. The content of the next theorem is that this situation cannot arise when the total quotient ring is a 0-dimensional ring.

THEOREM 4. Let $R$ be a ring with 0-dimensional total quotient ring, then the following are equivalent:

(a) $R$ admits at least one chained overring;

(b) $T(R)$ is a chained ring; and

(c) Every valuation overring of $R$ is a chained ring.

Proof. $\quad(\mathrm{a}) \Rightarrow(\mathrm{b})$ : Let $V$ be a chained overring of $R$, then $T(V)=$ $T(R)$ and by Corollary $3 T(R)$ is a chained ring.

$(b) \Rightarrow(c)$ : If $V$ is a valuation overring of $R$, let $T=T(V)=$ $T(R)$. Since $T$ is 0 -dimensional, we have that $Z(T)=N(T)$ [the nil radical of $T$ ]. Since $z$ in $Z(T)$ implies that there is an integer $n>1$ such that $z^{n}=0$, then $z$ is integral over $V$, and therefore $z$ is in $V$ since $V$ is integrally closed. Thus $Z(T) \subseteq V$, and we have that $V$ is a chained ring.

(c) $\Rightarrow(a)$ : Obvious.

A ring $R$ is a von Neumann regular ring if for each $r$ in $R$, there is a $b$ in $R$ such that $r^{2} b=r$. von Neumann regular rings are 0 -dimensional rings and have no nonzero nilpotents.

COROLlaRY 4. If $R$ admits a chained overring, then $T(R)$ is 
von Neumann reqular if and only if $R$ is an integral domain.

We characterize rings in which every valuation overring is a chained ring.

THEOREM 5. Let $R$ be ring with total quotient ring T. Let $\bar{R}$ be the integral closure of $R$ in T. Every valuation overring of $R$ is a chained ring if and only if $Z(T)$ is the conductor of $\bar{R}$ in $T$.

Proof. $(\Rightarrow)$ : Let $V$ be an arbitrary valuation overring of $R$, then $V$ is a chained ring. Clearly, $Z(T)=Z(V)$ is the conductor of $V$ in $T$. Therefore, $Z(R)=Z(T) \cap R$, and $R$ is an additively regular ring. This implies that $\bar{R}$ is the intersection of a family of valuation rings $\left\{V_{a}\right\}$. So, $Z(T) \subseteq \bigcap V_{a}=\bar{R}$.

$(\Longleftrightarrow)$ : If $Z(T)$ is the conductor of $\bar{R}$ in $T$, then $Z(T)$ is the unique maximal ideal of $T$ and thus is a prime ideal. Hence, $Z(R)=Z(T) \cap R$ is a prime ideal of $R$, and thus $R$ is additively regular. Whence $\bar{R}=\bigcap V_{a}$ where $\left\{V_{a}\right\}$ is the set of valuation rings between $R$ and $T(R)$. Therefore, $Z(T) \subseteq V_{a}$ for all $a$ and hence each $V_{a}$ is a chained ring.

Our final result is of interest in the light that it extends the theory of chained rings although it is not directly concerned with overrings. It gives necessary and sufficient conditions for a Prüfer valuation ring to be a chained ring.

Let $P$ be a prime ideal of a ring $R$. The core of $P$, denoted by $C(P)$, is the set of all elements $b$ in $R$ such that for each regular $r$ in $R$, there exists an element $e$ in $R \backslash P$ such that be/r is in $R$. For a valuation ring $V$ with $M=\{x \in T(V) \mid v(x)>0\}$, the core of $M$ is the prime ideal of zero for which the valuation, $v$, is infinite.

THEOREM 6. Let $V$ be a valuation ring with a unique maximal regular ideal $M$. Then $V$ is a chained ring if and only if the following three conditions hold:

(a) $V$ is a Prüfer ring;

(b) $C(M)=Z(V)$;

(c) $a, b \in C(M)$ implies that $a$ divides $b$ or $b$ divides $a$ in $V$.

Proof. $(\Rightarrow)$ : The fact that chained rings are Prüfer is trivial, since finitely generated regular ideals are principal ideals generated by regular elements. Theorem 2 tells us that

$$
C(M)=\{x \in V \mid v(x)=\infty\}=Z(V) .
$$


And certainly (c) is true.

$(\Leftarrow)$ : If $a$ and $b$ are in $V$, we must show that $a$ divides $b$ or $b$ divides $a$ in $V$. This follows from the hypotheses, if $a$ and $b$ are in $C(M)$; and by the quasi-valuation property, if $a$ and $b$ are regular. Assume that $a$ is regular and that $b$ is a zero divisor. Then $b / a$ is in $T(V)$ and $v(b / a)=v(b)-v(a)$. But $v(b)=\infty$ and $v(a)<\infty$. Thus, $v(b / a)=\infty$ which implies that $b / a$ is in $V$, or that $a$ divides $b$ in $V$.

\section{REFERENCES}

1. M. Boisen, and M. Larsen, Prüfer and valuation rings with zero divisors, Pacific J. Math., 40 (1972), 7-12.

2. - On Prïfer rings as images of Prüfer domains, Proc. Amer. Math. Soc., 40 (1973), 87-90.

3. E. Davis, Overrings of commutative rings II. Integrally closed overrings, Trans. Amer. Math. Soc., 110 (1964), 196-212.

4. R. Gilmer, On Prüfer rings, Bull. Amer. Math. Soc., 78 (1972), 223-224.

5. - Multiplicative Ideal Theory. Queens Papers on Pure and Applied Mathematics, Vol. 12. Kingston, Ontario, Canada: Queens University Press, 1968.

6. R. Gilmer, and J. Huckaba, 4-rings, J. Algebra, 28 (1974), 414-432.

7. M. Griffin, Prüfer rings with zero divisors, J. Reine Angew. Math., 239/240 (1970), $55-67$

8. J. Huckaba, On valuation rings that contain zero divisors, Proc. Amer. Math. Soc., 40 (1973), 9-15.

9. T. Hungerford, On the structure of principal ideal rings, Pacific J. Math., 25 (1968), 543-547.

10. M. Larsen and P. McCarthy, Multiplicative Theory of Ideals, New York, N. Y.: Academic Press, 1971.

11. M. Manis, Valuations on a commutative ring, Proc. Amer. Math. Soc., 20 (1969), 193-198.

Received May 2, 1975 and in revised form January 27, 1976. This paper is part of the author's Ph. D. thesis written under the direction of James A. Huckaba at the University of Missouri-Columbia.

SaINT Mary's College

Current Address: Saint Mary's College

Winona, Minnesota 55987 



\section{PACIFIC JOURNAL OF MATHEMATICS}

\section{EDITORS}

RICHARD ARENS (Managing Editor) University of California

Los Angeles, California 90024

R. A. Beaumont

University of Washington

Seattle, Washington 98105
J. DUGUNDJI

Department of Mathematics University of Southern Californı

Los Angeles, California 90007

D. Gilbarg and J. Milgram

Stanford University

Stanford, California 94305

\section{ASSOCIATE EDITORS}
E. F. BECKENBACH
B. H. NeumanN
F. WOLF
K. YosHIDA

\section{SUPPORTING INSTITUTIONS}

UNIVERSITY OF BRITISH COLUMBIA

CALIFORNIA INSTITUTE OF TECHNOLOGY

UNIVERSITY OF CALIFORNIA

MONTANA STATE UNIVERSITY

UNIVERSITY OF NEVADA

NEW MEXICO STATE UNIVERSITY

OREGON STATE UNIVERSITY

UNIVERSITY OF OREGON

OSAKA UNIVERSITY
UNIVERSITY OF SOUTHERN CALIFORNIA

STANFORD UNIVERSITY

UNIVERSITY OF TOKYO

UNIVERSITY OF UTAH

WASHINGTON STATE UNIVERSITY

UNIVERSITY OF WASHINGTON

* * * *

AMERICAN MATHEMATICAL SOCIETY

NAVAL WEAPONS CENTER 


\section{Pacific Journal of Mathematics \\ Vol. 65, No. $1 \quad$ September, 1976}

David Lee Armacost, Compactly cogenerated LCA groups ............. 1

Sun Man Chang, On continuous image averaging of probability measures ...... 13

J. Chidambaraswamy, Generalized Dedekind $\psi$-functions with respect to a

polynomial. II................................... 19

Freddy Delbaen, The Dunford-Pettis property for certain uniform algebras ..... 29

Robert Benjamin Feinberg, Faithful distributive modules over incidence

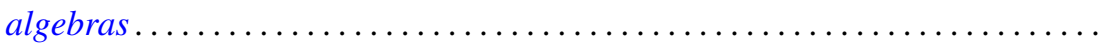

Paul Froeschl, Chained rings . . . . . . . . . . . . . . . . . . . .

John Brady Garnett and Anthony G. O'Farrell, Sobolev approximation by a sum

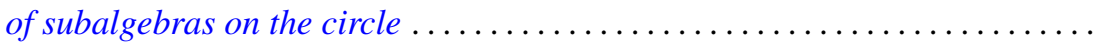

Hugh M. Hilden, José M. Montesinos and Thomas Lusk Thickstun, Closed

oriented 3-manifolds as 3-fold branched coverings of $S^{3}$ of special type.....

Atsushi Inoue, On a class of unbounded operator algebras ................

Peter Kleinschmidt, On facets with non-arbitrary shapes.

Narendrakumar Ramanlal Ladhawala, Absolute summability of Walsh-Fourier

series

Howard Wilson Lambert, Links which are unknottable by maps . . . . . . . . . . .

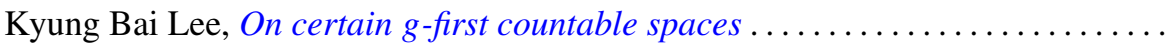

Richard Ira Loebl, A Hahn decomposition for linear maps .................

Moshe Marcus and Victor Julius Mizel, A characterization of functionals on $W_{1}^{p}$ possessing autonomous kernels. I . .

James Miller, Subordinating factor sequences and convex functions of several

variables.

Keith Pierce, Amalgamated sums of abelian l-groups ...

Jonathan Rosenberg, The $C^{*}$-algebras of some real and $p$-adic solvable

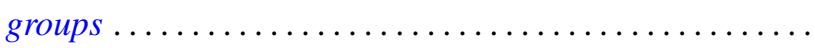

Hugo Rossi and Michele Vergne, Group representations on Hilbert spaces defined

in terms of $\partial_{b}$-cohomology on the Silov boundary of a Siegel domain . .

Mary Elizabeth Schaps, Nonsingular deformations of a determinantal

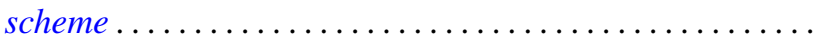

S. R. Singh, Some convergence properties of the Bubnov-Galerkin method...

Peggy Strait, Level crossing probabilities for a multi-parameter Brownian

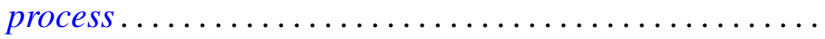

Robert M. Tardiff, Topologies for probabilistic metric spaces .

Benjamin Baxter Wells, Jr., Rearrangements of functions on the ring of integers of

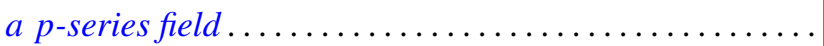

Robert Francis Wheeler, Well-behaved and totally bounded approximate identities for $C_{0}(X)$.

Delores Arletta Williams, Gauss sums and integral quadratic forms over local

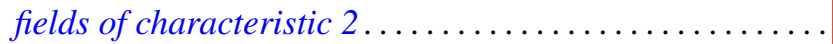

John Yuan, On the construction of one-parameter semigroups in topological 